\title{
Distribuição de fertilizantes em um sistema de fertigação por gotejamento
}

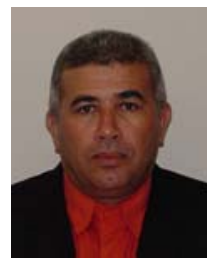

Valdemício F. de Sousa' ${ }^{1}$, Marcos V. Folegatti ${ }^{2}$, José A. Frizzone ${ }^{3}$, Rodrigo A. de L. Corrêa ${ }^{4} \&$ Cristina M. Alencar ${ }^{5}$

\footnotetext{
1 Embrapa Meio-Norte. Av. Duque de Caxias 5650, Buenos Aires, CEP 64006-220, Teresina, PI. Fone: (86) 2251141. E-mail: vfsousa@cpamn.embrapa.br (Foto)

2 ESALQ/USP. CEP 13418-900, Piracicaba, SP. Fone: (19) 3429 4217. E-mail: mvfolega@carpa.ciagri.usp.br

${ }^{3}$ ESALQ/USP. Fone: (19) 3429 4217. E-mail: frizzone@carpa.ciagri.usp.br

${ }^{4}$ ESALQ/USP. Fone: (19) 3429 4217. E-mail: ralcorre@carpa.ciagri.usp.br

${ }^{5}$ FCA/UNESP. CEP 18603-970, Botucatu, SP. Fone: (14) 6802 7165. E-mail: calencar@fca.unesp.br
}

Protocolo 72 - 20/5/2002 - Aprovado em 11/2/2003

\begin{abstract}
Resumo: Objetivou-se, com este trabalho, avaliar a uniformidade de distribuição temporal e espacial de fertilizantes em um sistema de fertirrigação por gotejamento, instalado numa área cultivada com maracujazeiro amarelo, em Piracicaba, SP. O sistema de irrigação utilizado foi por gotejamento, com emissores de vazão de 2,4 $\mathrm{L} \mathrm{h}^{-1}$. A fertirrigação foi realizada com uma bomba injetora de fertilizante de acionamento hidráulico e o tempo de injeção da solução no sistema foi de 1,0 h. Para se avaliar a uniformidade de distribuição de nutrientes nas linhas de irrigação, coletaram-se amostras da solução nas linhas laterais aos 2, 12, 22, 32, 42 e 52 min após o início da aplicação dos fertilizantes, nos gotejadores da primeira planta $(2 \mathrm{~m})$, e nos das plantas localizadas a $1 / 3(10 \mathrm{~m}), 2 / 3(22 \mathrm{~m})$ e da última planta $(34 \mathrm{~m})$. A concentração de fertilizantes nas amostras foi determinada através da condutividade elétrica e a uniformidade de distribuição de fertilizantes avaliada pela relação entre os seus menores valores de condutividade elétrica e a média das amostras. Nas linhas lavadas antes da fertirrigação, os índices de uniformidade apresentaram-se maiores e se observou que a uniformidade de distribuição de fertilizantes aumentou com o tempo até os 42 min e se manteve constante a partir desse instante.
\end{abstract}

Palavras-chave: coeficiente de uniformidade, condutividade elétrica, uniformidade de irrigação

\section{Fertilizer distribution in a drip fertigation system}

\begin{abstract}
The aim of this study was to evaluate the spatial and temporal fertilizer distribution uniformity in a drip fertigation system, installed in an experimental area with passion fruit crop in Piracicaba, SP, Brazil. The discharge of emitter was $2.4 \mathrm{~L} \mathrm{~h}^{-1}$ and a fertilizer injector pump of hydraulic action was used. The injection time of solution was of one hour. To evaluate the uniformity of nutrients distribution samples of solution were collected 2, 12, 22, 32, 42, and 52 min after the start of fertigation in the emitters from the first plant $(2 \mathrm{~m})$ and in the emitters localized at $1 / 3(10 \mathrm{~m})$ and 2/3 $(22 \mathrm{~m})$ distance of lateral line length and those installed on last plant $(34 \mathrm{~m})$ of each lateral line. The fertilizer concentration in the samples was determined by the electrical conductivity of the solutions. The relation between the minimum and the mean electrical conductivity values was used to evaluate the uniformity of fertilizer distribution. The distribution uniformity indexes in the lines washed before the fertigation were higher compared to others. It was observed that the uniformity increased with time until 42 min after the injection and maintained constant thereafter.
\end{abstract}

Key words: uniformity coefficient, electrical conductivity, irrigation uniformity

\section{INTRODUÇÃO}

A aplicação de fertilizantes através da água de irrigação proporciona aumento da eficiência da adubação e do uso de nutrientes pela cultura, devido à possibilidade do parcelamento da aplicação, conforme as necessidades da cultura (Dasberg \& Bresler, 1985). Todavia, os fertilizantes devem ser distribuídos uniformemente com a água de irrigação em toda a área, ou ao longo das linhas de cultivo (Sousa \& Sousa, 1993). Isto requer que o sistema de irrigação seja dimensionado para funcionar com alto índice de uniformidade de distribuição de água (Sousa et al., 1998).

Na irrigação localizada a uniformidade de vazão é função do comprimento da linha lateral, da pressão de entrada, do 
espaçamento entre os emissores e da vazão do fluxo total (Wu \& Gitlin, 1974). Para Howell \& Hiller (1974) a variação da vazão está relacionada também com as perdas de pressão por atrito ao longo da tubulação e nas inserções dos emissores, ganho ou perda de energia de posição, qualidade da matéria-prima e dos processos de fabricação, obstruções e efeito da temperatura da água sobre o regime de escoamento, além da geometria dos emissores.

Fatores como a concentração da solução a ser injetada, tempo de aplicação, solubilidade e pureza dos produtos, afetam também a uniformidade de distribuição de nutrientes, comprometendo o rendimento da cultura (Grobbelaar \& Lourens, 1974; Holman, 1978; Rolston et al., 1979). Zanini (1987), Alves (1992) e Sampaio et al. (1997) constataram que, no início da fertirrigação, as concentrações de fertilizantes foram maiores nos primeiros emissores, o que se atribui ao tempo que a solução nutritiva gasta para chegar aos emissores finais e estabilizar a concentração.

Uma boa uniformidade de distribuição de água de irrigação é fundamental para um bom manejo da fertirrigação. Entretanto, a uniformidade de distribuição de nutrientes pode ser também afetada pela variação da concentração desses na água de irrigação, a qual está relacionada com: o grau de agitação ou mistura da solução no tanque, a variação da taxa de injeção da solução e a variação da vazão no sistema de irrigação durante a fertirrigação (Rolston et al., 1979; Burt et al., 1998).

O manejo adequado da fertirrigação requer que a injeção de fertilizantes seja iniciada quando toda tubulação estiver cheia de água e os emissores em pleno funcionamento; caso contrário, a uniformidade de distribuição de fertilizantes será prejudicada. Neste procedimento, o tempo de fertirrigação é muito importante na uniformidade de distribuição dos fertilizantes em toda a área cultivada, o qual divide a aplicação em três etapas: a primeira para enchimento da tubulação, a segunda para aplicação propriamente dita da solução na água de irrigação e a terceira para promover a lavagem da tubulação e dos emissores, tempo este que raramente deverá ser inferior a 30 min (Frizzone et al., 1985); todavia, como a estabilização da concentração de fertilizantes nas linhas de irrigação ocorre após 20 min (Sampaio et al., 1997) maior tempo de fertirrigação conduz a uma uniformidade melhor de distribuição de fertilizantes na área (Zanini, 1987).

Para que ocorra aplicação uniforme em toda a área, Holman (1978) sugere que é preferível injetar-se, em quase todos os casos, a solução, lentamente, ao sistema de irrigação; aplicação muito rápida pode distribuir fertilizante apenas em uma parte da área. Em sistemas de irrigação por gotejamento, Globbelaar \& Lourens (1974) indicam que para se obter melhor uniformidade de distribuição, o tempo de injeção de fertilizantes deve ser de 3 a 5 h.

A avaliação da uniformidade de distribuição de nutrientes em sistemas de irrigação, requer a coleta de água e, posteriormente, determina-se a concentração do elemento na amostra. Devido as dificuldades na determinação de alguns elementos, estudos têm avaliado a uniformidade de distribuição de nutrientes através da condutividade elétrica, como indicador da concentração, porém os elementos não são quantificados.
O objetivo deste trabalho foi avaliar a uniformidade de distribuição espacial e temporal de fertilizantes em um sistema de fertirrigação por gotejamento, instalado em uma área experimental cultivada com maracujazeiro.

\section{MATERIAL E MÉTODOS}

O trabalho foi conduzido no Campus Experimental da Fazenda Areão, pertencente ao Departamento de Engenharia Rural ESALQ - USP, localizada em Piracicaba, SP (22 42' 30'S e $\left.47^{\circ} 30^{\prime} 00^{\prime \prime} \mathrm{W}\right)$. A área experimental com a cultura do maracujá amarelo foi constituída de 4 blocos com 5 linhas de 9 plantas espaçadas $4 \mathrm{~m}$.

O sistema de irrigação utilizado foi gotejamento com emissores autocompensante e autolimpante com vazão de $2,4 \mathrm{~L} \mathrm{~h}^{-1}$. As linhas laterais, com diâmetro de $0,012 \mathrm{~m}$ e comprimento de $36 \mathrm{~m}$, foram distribuídas ao lado das plantas e, para cada uma destas, colocaram-se dois gotejadores espaçados $0,60 \mathrm{~m}$.

A fertirrigação foi realizada com uma bomba injetora de fertilizante de acionamento hidráulico. O tempo de injeção da solução no sistema foi de $1,0 \mathrm{~h}$. Aplicou-se potássio $(\mathrm{KCl})$ com condutividade elétrica (CE) da solução no tanque de $16,16 \mathrm{dS} \mathrm{m}^{-1}$, nas linhas laterais $\mathrm{L}_{1}, \mathrm{~L}_{2}, \mathrm{~L}_{3} \mathrm{e} \mathrm{L}_{4}$. As linhas laterais $\mathrm{L}_{1}$ (final da área) e $\mathrm{L}_{3}$ (início da área) foram submetidas a lavagem após a última fertirrigação, enquanto $\mathrm{L}_{2}$ (final da área) e $\mathrm{L}_{4}$ (início da área) não receberam o mesmo tratamento.

Os testes foram realizados após quatro meses de funcionamento do sistema e, para se avaliar a uniformidade de distribuição espacial e temporal de nutrientes nas linhas de irrigação coletaram-se amostras da solução nas linhas laterais, nos gotejadores da primeira planta $(2 \mathrm{~m})$, e nos das plantas localizadas a 1/3 (10 m), 2/3 (22 m) e da última planta (34 m). As amostras foram coletadas aos 2, 12, 22, 32, 42 e 52 min após o início da aplicação dos fertilizantes e a concentração de fertilizantes nas amostras foi determinada através da condutividade elétrica.

Os valores de CE foram plotados em gráficos tipo superfície do Surffer e, para se determinar a uniformidade de distribuição de potássio utilizou-se o método descrito em Lopez et al. (1992) que relaciona os menores valores e a média das amostras.

$$
\mathrm{CU}=\frac{\mathrm{CEmi}}{\mathrm{CEm}} 100
$$

em que:

CU - coeficiente de uniformidade de distribuição da condutividade elétrica (\%)

CEmi - menor valor de condutividade elétrica $\left(\mathrm{dS} \mathrm{m}^{-1}\right)$

$\mathrm{CEm}$ - condutividade elétrica média $\left(\mathrm{dS} \mathrm{m}^{-1}\right)$

\section{RESULTADOS E DISCUSSÃO}

Para as linhas laterais $\mathrm{L}_{1}$ e $\mathrm{L}_{3}$, nas amostragens realizadas aos 2 min após o início da injeção de fertilizantes, constataramse valores de $\mathrm{CE}$ inferiores a $1 \mathrm{dS} \mathrm{m}^{-1}$ ao longo de toda a linha. Nas amostragens seguintes, os valores de CE aumentaram com o tempo, tendendo à estabilização da concentração aos 22 min 
(Figuras 1 e 2). Os valores de $\mathrm{CE}$ registrados em $\mathrm{L}_{1}$ foram menores que os obtidos em $\mathrm{L}_{3}$, na ordem de $40,35,49,54,5 \mathrm{e}$ $82 \%$ nas coletas realizadas aos $12,22,32,42$ e $52 \mathrm{~min}$, respectivamente. Essas variações da concentração na água de irrigação entre $\mathrm{L}_{1}$ e $\mathrm{L}_{3}$ podem ser atribuídas à distância dessas linhas laterais em relação ao ponto de injeção de fertilizantes. Pontos mais distantes tendem a receber menores quantidades de fertilizantes e, conseqüentemente, as concentrações serão menores.

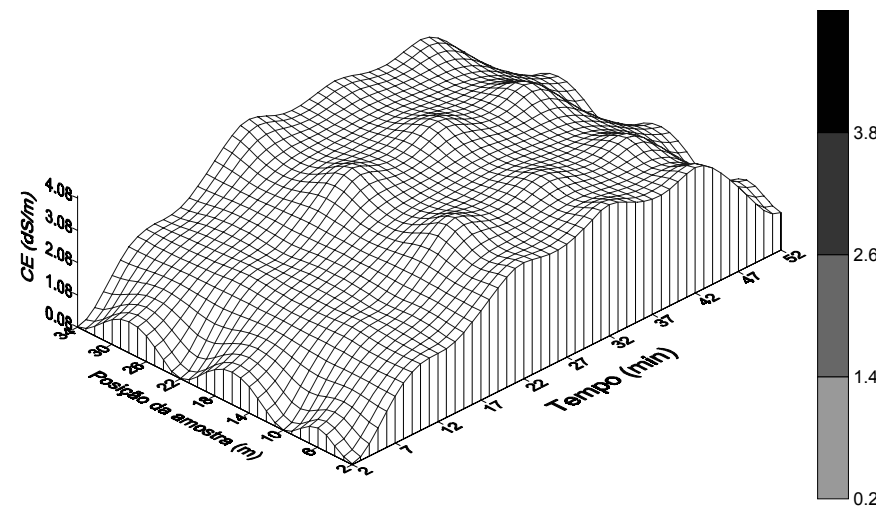

Figura 1. Distribuição espacial e temporal de potássio em linhas laterais de irrigação por gotejamento, quando submetidas a lavagem antes da fertirrigação. Linha lateral $\left(\mathrm{L}_{1}\right)$ localizada no final da área

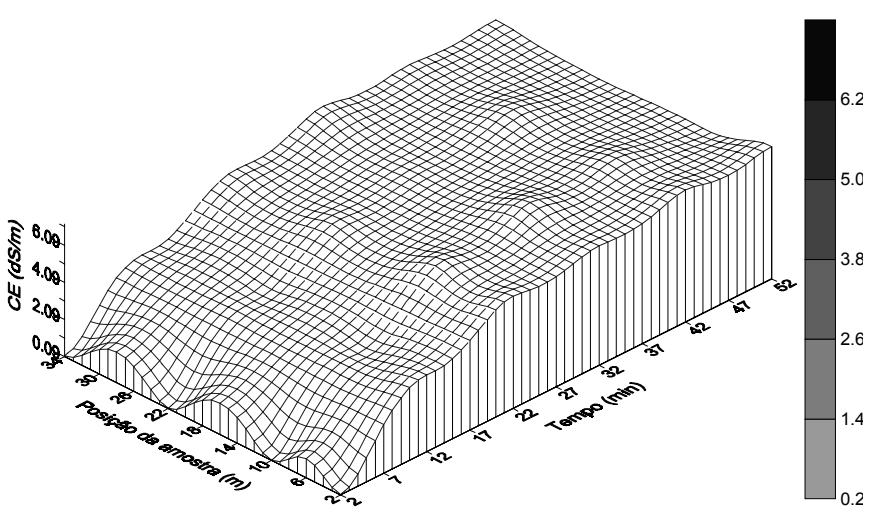

Figura 2. Distribuição espacial e temporal de potássio em linhas laterais de irrigação por gotejamento, quando submetidas a lavagem antes da fertirrigação. Linha lateral $\left(\mathrm{L}_{3}\right)$ localizada no início da área

Para as linhas laterais $\mathrm{L}_{2}$ e $\mathrm{L}_{4}$, os valores de CE nas amostragens realizadas aos $2 \mathrm{~min}$ aumentaram do início para o final da linha, sendo mais acentuados na linha lateral $\mathrm{L}_{2}$, que variaram de forma crescente, entre 0,60 e $6,57 \mathrm{dS} \mathrm{m}^{-1}$, enquanto $\mathrm{em} \mathrm{L}_{4}$ a variação foi entre $0,46 \mathrm{e} 1,21 \mathrm{dS} \mathrm{m}^{-1}$. Este comportamento diverge do que em geral ocorre em fertirrigação, onde quase sempre no início da aplicação os menores valores na concentração ocorrem nos emissores localizados no final da linha lateral, tal como foi constatado por Zanini (1987), Alves (1992) e Sampaio (1995). Esta divergência se deveu ao acúmulo de resíduos de fertilizantes provenientes de aplicações anteriores, uma vez que essas laterais não foram submetidas a lavagem após as duas últimas aplicações e o regime de fluxo laminar de escoamento de água ocorrido, após desligar o sistema de irrigação, provoca sedimentação no final da linha, como mencionado por Rolston et al. (1979).

$\mathrm{Na}$ linha lateral $\mathrm{L}_{2}$, localizada a $35 \mathrm{~m}$ do ponto de injeção de fertilizantes, registrou-se redução nos valores da condutividade elétrica nas coletas realizadas aos $12 \mathrm{~min}$ (Figura 3 ) a qual pode estar relacionada ao efeito espacial na distribuição de fertilizantes. A posição desta lateral na área possibilita aos fertilizantes levarem mais tempo para chegar e se estabilizarem, quando comparado com as laterais localizadas mais próximo do ponto de injeção.

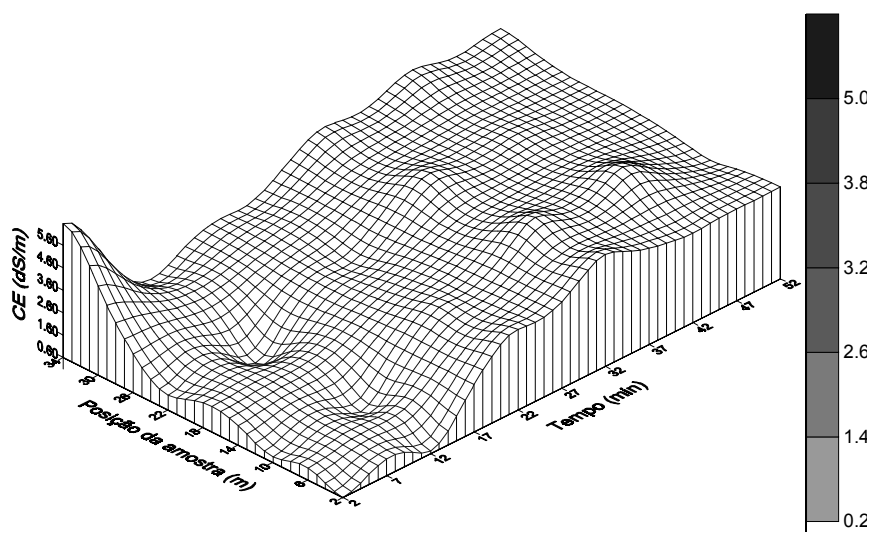

Figura 3. Distribuição espacial e temporal de potássio em linhas laterais de irrigação por gotejamento, quando submetidas a lavagem antes da fertirrigação. Linha lateral $\left(\mathrm{L}_{2}\right)$ localizada no final da área

No que se refere à estabilização da concentração, constatou-se que a lateral $\mathrm{L}_{4}$ (Figura 4 ) tendeu a estabilizar-se a partir dos 22 min e com redução acentuada aos 52 min, enquanto para $\mathrm{L}_{2}$ a estabilização foi notada a partir dos $32 \mathrm{~min}$, com ligeira redução ao 52 min. Esses resultados estão de acordo com Zanini (1987) e Sampaio et al. (1997) e evidenciam que a aplicação de fertilizantes em tempo inferior a $1 \mathrm{~h}$ pode promover uma aplicação desuniforme na área, tal como destacam Globbelaar \& Lourens (1974).

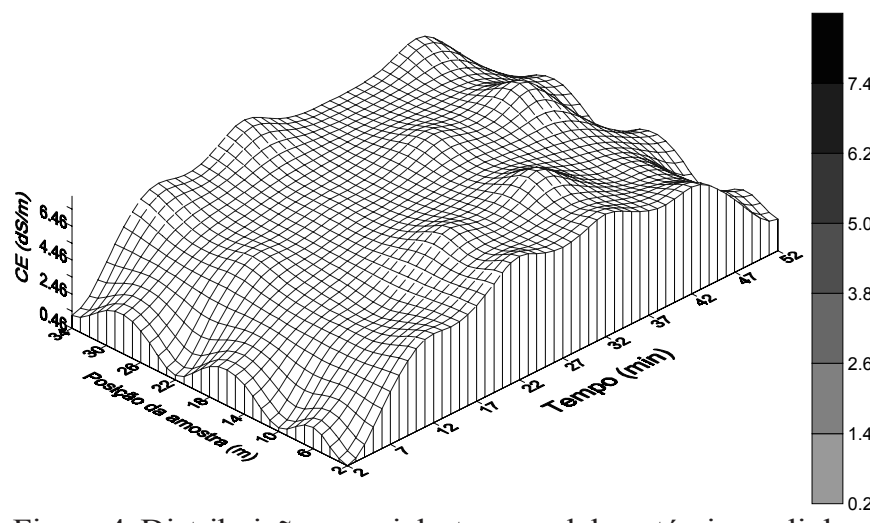

Figura 4. Distribuição espacial e temporal de potássio em linhas laterais de irrigação por gotejamento, quando submetidas a lavagem antes da fertirrigação. Linha lateral $\left(\mathrm{L}_{4}\right)$ localizada no início da área

As variações na distribuição de fertilizantes entre emissores na mesma linha e entre os tempos de amostragens, avaliadas 
pelo coeficiente de variação $(\mathrm{CV})$ mostraram que, espacialmente, essas variações foram maiores, com CV entre 35,56 e $64,67 \%$, que as registradas no tempo (Tabelas 1 e 2 ). No caso da variação temporal, constataram-se menores valores de $\mathrm{CV}$ nas laterais $\mathrm{L}_{1}(3,13$ a $8,31 \%)$ e $\mathrm{L}_{3}(4,39$ a $19,47 \%)$ enquanto para $\mathrm{L}_{2}$ e $\mathrm{L}_{4}$, os valores de $\mathrm{CV}$ foram maiores, principalmente nas amostragens realizadas aos 2 e $12 \mathrm{~min}$.

Tabela 1. Valores de coeficientes de variação (CV\%) na distribuição de fertilizantes, ao longo das linhas laterais de gotejamento

\begin{tabular}{|c|c|c|c|c|c|}
\hline \multirow{2}{*}{$\begin{array}{l}\text { Linhas } \\
\text { Laterais }\end{array}$} & \multicolumn{4}{|c|}{ Posição da Planta na Linha Lateral (m) } & \multirow{2}{*}{ Média } \\
\hline & 2 & 10 & 22 & 34 & \\
\hline $\mathrm{L}_{1}$ & 64,64 & 65,53 & 64,87 & 64,70 & 64,93 \\
\hline $\mathrm{L}_{2}$ & 63,89 & 62,56 & 59,89 & 35,56 & 55,47 \\
\hline $\mathrm{L}_{3}$ & 64,64 & 65,53 & 64,87 & 64,69 & 64,93 \\
\hline $\mathrm{L}_{4}$ & 59,35 & 63,59 & 57,08 & 51,04 & 57,76 \\
\hline Media & 60,05 & 61,56 & 58,83 & 51,24 & 57,92 \\
\hline
\end{tabular}

Tabela 2. Valores de coeficiente de variação (CV\%) na distribuição de fertilizantes em linhas laterais de gotejamento, nos respectivos tempos de amostragem

\begin{tabular}{cccccccc} 
Linhas & \multicolumn{7}{c}{ Tempo de Amostragem (min) } \\
\cline { 2 - 7 } Laterais & 2 & 12 & 22 & 32 & 42 & 52 & \\
\hline $\mathrm{L}_{1}$ & 8,31 & 4,32 & 3,70 & 4,61 & 3,14 & 4,49 & 4,8 \\
$\mathrm{~L}_{2}$ & 123 & 59,7 & 14,9 & 5,26 & 11,4 & 10,3 & 37,4 \\
$\mathrm{~L}_{3}$ & 19,8 & 9,73 & 5,89 & 6,48 & 4,36 & 6,89 & 8,8 \\
$\mathrm{~L}_{4}$ & 41,6 & 14,2 & 10,8 & 11,3 & 14,1 & 13,9 & 17,7 \\
\hline Média & 48,0 & 22,0 & 8,84 & 6,92 & 8,26 & 8,89 & 17,2 \\
\hline
\end{tabular}

Uma análise nos valores de coeficientes de uniformidade (CU) de distribuição temporal de fertilizantes (Tabela 3) confirma a tendência mostrada pelo CV. Os maiores valores de CU (95,9 e 94,3\%) foram registrados nas laterais $\mathrm{L}_{1}$ e $\mathrm{L}_{3}$, respectivamente. Para as linhas laterais $\mathrm{L}_{2}$ e $\mathrm{L}_{4}$, no início da fertirrigação, a distância do ponto de injeção influenciou nos valores de CU, pois os menores valores $(25,7$ e $54,1 \%)$ foram obtidos em $\mathrm{L}_{2}$, que se localizava mais distante do ponto de injeção do $\mathrm{L}_{4}$.

Tabela 3. Valores de coeficientes de uniformidade de distribuição de fertilizantes (CU\%) em linhas laterais de gotejamento, nos respectivos tempos de amostragem

\begin{tabular}{cccccccc} 
Linhas & \multicolumn{7}{c}{ Tempo de Amostragem (min) } \\
\cline { 2 - 7 } Laterais & 2 & 12 & 22 & 32 & 42 & 52 & \\
\hline $\mathrm{L}_{1}$ & 88,9 & 94,4 & 94,9 & 93,6 & 95,9 & 94,6 & 93,7 \\
$\mathrm{~L}_{2}$ & 25,7 & 64,1 & 84,6 & 93,4 & 86,0 & 89,2 & 73,8 \\
$\mathrm{~L}_{3}$ & 81,1 & 94,3 & 94,1 & 91,6 & 94,1 & 94,1 & 91,5 \\
$\mathrm{~L}_{4}$ & 59,4 & 81,7 & 84,0 & 84,7 & 81,0 & 81,1 & 78,7 \\
\hline Média & 63,8 & 83,6 & 89,4 & 90,8 & 89,3 & 89,7 & 84,4 \\
\hline
\end{tabular}

\section{CONCLUSÕES}

1. Nas linhas laterais lavadas antes da fertirrigação, a uniformidade de distribuição de fertilizantes foi maior que nas demais.

2. A uniformidade de distribuição de fertilizantes aumentou com o tempo, até os 22 min e se manteve constante a partir desse instante.
3. A distribuição temporal de fertilizantes foi maior que a espacial verificada ao longo das linhas laterais.

\section{LITERATURA CITADA}

Alves, D.N.B. Desempenho da bomba injetora e do tanque de derivação de fluxo na aplicação de cloreto de potássio em microaspersão. Lavras: Escola Superior de Agricultura de Lavras, 1992, 72p. Dissertação Mestrado

Burt, C.; O’Connor, K.; Ruehr, T. Fertigation. San Luis Obispo: California Polytechnic State University. 1998. p.53-61.

Dasberg, S.; Bresler, E. Drip irrigation manual. Tel Avive: International Irrigation. Information Center. 1985, 85p.

Frizzone, J.A.; Zanini, J.R.; Paes, L.A.D.; Nascimento, V.M. Fertirrigação mineral. Ilha Solteira: UNESP, 1985.31p. Boletim Técnico, 2

Globbelaar, H.L.; Lourens, F. Fertilizer applications with drip irrigation. In: International Drip Irrigation Congress. Proceedings... San Diego, p. 405-410, 1974.

Holman, H. Fertilizer and chemical injection for irrigation systems. Citrus \& Vegetable Magazine, Tampa, v.41, n.7, p.46-48, 1978.

Howell, T.A.; Hiller, E.A. Designing trickle irrigation laterals for uniformity. In: International Drip Irrigation Congress, 2, Proceedings... Riverside, p.299-304, 1974.

Lopez, J.R.; Abreu, J.M.H.; Regalado, A.P.; Hernadez, J.F.G. Riego localizado. 2 ed. Madrid: Centro Nacional de Tecnologia de Regadios, 1992. p.217-229.

Rolston, D.E.; Rauschkolb, R.S.; Phene, C.; Miller, R.J.; Uriu, K.; Carlso, R.M.; Handerson, D.W. Applying nutrients and other chemicals to trickle-irrigated crops. Derley: University of California, Division of Agricultural Science, Bulletin, 1981, $14 p$.

Sampaio, S.C. Distribuição do cloreto de potássio aplicado em linha de gotejamento por bomba injetora e tanque de derivação de fluxo. Lavras: Escola Superior de Agricultura de Lavras, 1995, 85p. Dissertação Mestrado

Sampaio, S.C.; Faria, M.A. de; Lima, L.A.; Oliveira, M.S. de; Silva, A.M. da. Distribuição espacial e temporal do cloreto de potássio aplicado em uma linha lateral de gotejamento por bomba injetora e tanque de derivação de fluxo. Engenharia Rural, Piracicaba, v.8, n.1, p.31-41, 1997.

Sousa, V.F. de; Folegatti, M.V.; Aragão, E.C.; Rebelo, A.L.F; Bastos, E.A. Avaliação de em um sistema de fertirrigação por microaspersão numa área cultivada com banana' In: Avances en el Manejo del Suelo y Agua en la Ingenieria Rural Latinoamericana. 1998, La Plata, 1998. CD-Rom

Sousa, V.F. de; Sousa, A. de P. Fertirrigação: tipos e seleção dos fertilizantes, aplicação e manejo. In: Congresso Brasileiro de Engenharia Agrícola, 22, 1993. Ilhéus. Anais... Ilhéus, SBEA, v.4, p.2529-2539, 1993.

Wu, I.P.; Gitlin, H.M. Drip irrigation design based on uniformity. Transactions of the ASAE, St. Joseph, v.17, n.1, p.429-432, 1974.

Zanini, J.R. Hidráulica da fertirrigação por gotejamento utilizando tanque de derivação de fluxo e bomba injetora. Piracicaba: ESALQ - USP, 1987, 103p. Tese Doutorado 